\title{
LOS CMS EN EL DESARROLLO DE ESTILOS DE APRENDIZAJE
}

\section{CMS IN THE DEVELOPMENT OF LEARNING STYLES}

\section{María Alejandrina Nivela Cornejo', Segundo Vicente Echeverría Desiderio², Marhilde Sánchez Villarroel de Gallardo ${ }^{3}$}

\author{
1,2 - Universidad de Guayaquil, Guayaquil, Ecuador
}

3 - Universidad de Zulia, Estado Zulia, Venezuela

1. Email: maria.nivelac@ug.edu.ec ORCID: http://orcid.org/0000-0002-0356-7243

2. Email: segundo.echeverriad@ug.edu.ec ORCID: http://orcid.org/0000-0002-0235-190X

3. Email: marsanchezg@gmail.com ORCID: https://orcid.org/0000-0001-5880-240X

Recibido: 11/05/2021 Aceptado: 21/07/2021

Para Citar: Nivela Cornejo , M. A., Segundo Vicente Echeverría Desiderio, S. V. E. D., \& Villarroel de Gallardo, M. S. . (2021). Los CMS en el desarrollo de estilos de aprendizaje. Revista Publicando, 8(31), 177-193. https://doi.org/10.51528/rp.vol8.id2243

\begin{abstract}
Resumen:
La presente investigación tuvo como objetivo analizar la gestión de contenidos en blogs, wikis, plataformas educativas, publicaciones periódicas y redes sociales por parte de estudiantes universitarios. Metodológicamente se realizó una investigación cuantitativa, de tipo descriptiva, con diseño no experimental, transeccional. La población fue de 150 estudiantes de la Universidad de Guayaquil, Facultad de Filosofía, Letras y Ciencias de la Educación, carrera Informática, se aplicó el muestreo no probabilístico por conveniencia, seleccionando a 50 estudiantes; se elaboró y aplicó un cuestionario cuya confiabilidad mediante el Alfa de Cronbach, fue 0,89. Para el análisis de datos se asumió la estadística descriptiva. Los resultados muestran una media muy alta en cuanto a la gestión de contenidos en blogs, plataformas educativas y redes sociales; alta en el caso de las wikis y media en publicaciones periódicas. Se concluye que en cuanto a funcionalidad, los estudiantes prefieren los gestores de contenido blogs y redes sociales.
\end{abstract}

Palabras clave: Blogs, Gestores de Contenido, Wikis, Plataformas Educativas, Publicaciones Periódicas, Redes Sociales.

\begin{abstract}
:
The present research aimed to analyze the content management in blogs, wikis, educational platforms, periodicals and social networks by university students. Methodologically, a quantitative, descriptive research was carried out, with a non-experimental, transectional design. The population was 150 students from the University of Guayaquil, Faculty of Philosophy, Letters and Education Sciences, Computer Science career, non-probability sampling was applied for convenience, selecting 50 students. A questionnaire was developed and applied; its reliability was 0.89 using Cronbach's Alpha. Descriptive statistics were assumed for data analysis. The results show a very high average in terms of content management in blogs, educational platforms and social networks; high in the case of wikis and media in periodicals. It is concluded that in terms of functionality, students prefer blogging and social media content managers.
\end{abstract}

Keywords: Blogs, Content Managers, Wikis, Educational Platforms, Periodicals, Social Networks. 


\section{INTRODUCCIÓN}

$\mathrm{E}$ 1 impacto alcanzado por la tecnología a nivel mundial en la educación conlleva a investigar y profundizar en un conjunto de problemas inherentes al proceso de aprendizaje y construcción del conocimiento. Ecuador no está fuera de este contexto. En los tiempos modernos se ha hecho indispensable el uso de nuevas herramientas digitales en la educación; por lo que es necesario conocer de qué manera los gestores de contenido pueden ayudar a generar conocimiento. No tiene sentido seguir desarrollando actividades pedagógicas con un desfase con la práctica y con el sistema social.

Celi (2018), plantea que se ha asumido muchas veces este conocimiento tecnológico como base del desarrollo social, debido a que brinda la posibilidad de resolver problemas y facilitar el modo de vida de las personas, considerando que puede satisfacer múltiples necesidades, tales como el intercambio de información y conocimiento dentro de las organizaciones, sobre todo en educación.

Es importante destacar que los estudiantes a nivel superior, mantienen su opinión de que el sistema educativo y sus estrategias son pretéritas, tomando en cuenta la existencia de un Sistema tradicional que utiliza herramientas obsoletas, las cuales les dificulta desarrollar eficazmente sus competencias académicas. Además de esto, los docentes consideran poco en sus estrategias las formas mediante las cuales el aprendiz, percibe y procesa la información; de aquí que los estilos de aprendizaje constituyen un factor importante en el resultado de aprendizaje.

En Ecuador, los profesores aún están en el proceso de adaptación a un sistema educativo que involucra la integración y actualización constante en el uso de TIC; y la rutina, el miedo y las dudas invaden su mente, hasta el punto de que su praxis está permeada por muchas interrogantes.

\section{Planteamiento del problema}

La ciencia y la tecnología, se consideran necesarias para lograr una sociedad donde sus habitantes tengan una mejor calidad de vida; por esta razón, cada vez existe una mayor ocupación por generar nuevos conocimientos, tecnologías e innovaciones para favorecer procesos de interacción humana y para ello es necesario hacer investigación, sobre todo a nivel educativo (Delgado, 2018).

Se concibe la educación como un proceso que le corresponde estar a la par de los cambios de la sociedad si aspira a alcanzar relevancia en el tiempo. En el pasado deben quedar los escenarios rígidos con prácticas predecibles, en la era moderna la sociedad de la información y del conocimiento conduce a plantear continuamente nuevas formas de enseñar y aprender, teniendo en cuenta modalidades y metodologías diversas, entre ellas la incorporación de las TIC y los servicios que ofrece la Web, tales como los gestores de contenido.

Así, al igual que en la primera generación 1.0 de la Web la forma de trabajar era unidireccional, estática, centralizada, secuencial, de solo lectura, y no interactiva; en la educación ocurría y todavía sucede algo similar, y es que los estudiantes acceden a las universidades o colegios, para ser educados por el profesor, el auténtico y único poseedor del conocimiento, haciendo referencia a la clase magistral como método predominante de enseñanza. 
Con la aparición de la Web 2.0, cambia la forma de percibir y utilizar la información en línea por parte de los usuarios, basada en las llamadas redes sociales, es dinámica, interactiva, de lectura y escritura, desarrolla la inteligencia colectiva y favorece el trabajo colaborativo, para ellos surgen los gestores de contenidos (CMS), los cuales permiten la creación y administración de contenidos en páginas web.

Esto ha exigido a la educación la interacción mediante roles más colaborativos, aunque todavía se está internamente realizando una enseñanza muy tradicional, es una etapa de transición; la verdadera revolución deberá venir de la mano de las posibilidades ilimitadas que ofrecen día a día las distintas tecnologías emergentes como la Web 3.0 y la Web 4.0; la primera (Web 3.0), conocida como web semántica porque utiliza de forma más eficiente de los datos, la data web, es interoperativa y el usuario tiene el control para hacer los cambios deseados, modificando directamente las bases de datos, está gestionada en la nube y puede ser ejecutada desde cualquier dispositivo con una alto grado de complejidad $y$ personalización.

En este contexto la educación deja de ser unidireccional para realizarse en múltiples direcciones, es decir, del profesor al estudiante, del estudiante al profesor, de estudiante a estudiante y a todo el mundo. La Web 4.0, como tecnología emergente está relacionada con la inteligencia artificial, el contenido es construido $y$ reconstruido en el contexto de forma constante y la educación es cambiada totalmente por una comunidad donde todos aprenden $y$ todos enseñan.
El desarrollo alcanzado por la informática a nivel mundial en diferentes áreas, en particular en la educación, plantea la necesidad de investigar y profundizar en un conjunto de problemas inherentes a la informática educativa, que tenga la flexibilidad de ajustarse y modificarse según el avance de las TIC. Esto implica que el problema planteado en esta investigación está inmerso dentro de un fenómeno globalizante en todas las áreas del conocimiento humano, especialmente en la tecnológica.

Ecuador no está fuera de este contexto, este efecto globalizante obliga al sistema educativo a colocarse a la par de otras regiones del mundo y requiere tener acceso a diversas herramientas digitales mediante el uso de internet. En los tiempos modernos se ha hecho indispensable el uso de nuevas herramientas digitales, debido al avance tecnológico acelerado que se está desarrollando en la sociedad y la educación ha sido influenciada por este cambio; por lo cual es muy necesario el acoplamiento de dichas herramientas y conocer de qué manera los gestores de contenido pueden ayudar a generar conocimiento en los estudiantes.

No tiene sentido seguir desarrollando actividades pedagógicas para formar un individuo para un mundo que ya no existe, pues ello expresaría un desfase con la práctica, provocando que no actúe y se retrotraiga en comportamientos que los excluye del sistema social de estos tiempos. Los estudiantes, sobre todo a nivel superior, mantienen su posición de que el sistema educativo y sus estrategias son caducas, por lo que su orientación profesional en muchos casos se ve frustrada, el hecho de mantener un sistema tradicional utilizando herramientas obsoletas no 
les permite desarrollar eficazmente sus competencias académicas. Ante tal situación surge la siguiente investigación, cuyo objetivo consistió en: analizar la Gestión de contenidos en blogs, wikis, plataformas educativas, publicaciones periódicas y redes sociales por parte de estudiantes universitarios.

\section{Gestores de contenido}

Existen diversas definiciones de este tema, sin embargo, debido a su forma de presentación sencilla y concreta, se escogió la ofrecida por García (2018), quien expresa que un gestor de contenidos, o traducido del inglés como Content Management System, llamado por sus siglas: CMS, se trata de una herramienta o recurso que favorece la creación y administración de contenidos mediante páginas de internet, es decir, la web.

Presenta como principal finalidad separar la interfaz gráfica de usuario (GUI) del contenido, con lo cual permite que personas sin conocimientos informáticos, puedan montar un sitio Web, sin nada que envidiar a los diseñados por ingenieros y analistas, incluso la mayoría de CMS traen secciones especializadas en noticias, foros, descargas, preguntas frecuentes, encuestas, galería de imágenes y quizás lo más importante control de usuarios mediante el uso de privilegios, contraseñas, manejo de grupos, permisos de lectura/escritura.

La mayoría de CMS son de libre distribución, bajo licencia GPL (Licencia Pública General de GNU o más conocida por su nombre en inglés GNU General Public Licenses) los puede descargar de Internet y utilizarlos sin ningún costo, por lo general se apoyan en grandes comunidades de software. La mayoría de CMS son desarrollados utilizando software libre, entre las herramientas más utilizadas para el desarrollo están:

- PHP como lenguaje de scripting.

- MYSQL como base de datos.

- Apache como servidor Web.

Existen muchas comunidades que desarrollan CMS de manera gratuita, entre las más importantes se pueden citar:

- Mambo http://www.mambo-foundation.org/

- PostNuke http://www.postnuke.org

- PHPNuke http://phpnuke.org

También existen alternativas para plataformas de software comercial como:

-ASPNuke http://www.aspnuke.com/

\section{PostNuke}

Es un CMS desarrollado plenamente en PHP, como base de datos utiliza MySQL y como servidor Web Apache, su principal ventaja es su diseño modular y la gran cantidad de módulos que se pueden encontrar en Internet, chat's, foros, descargas, noticias, galerías de imágenes o utilitarios. Con un solo clic se puede cambiar totalmente de apariencia un sitio PostNuke, incluso los usuarios pueden personalizar su manera de ver el sitio. Otra característica importante a ser destacada es el manejo multilingüe. Análogamente el CMS puede ser personalizado según las necesidades. Estas características lo hace un recurso que puede ser aprovechado en educación como un recurso con muchas potencialidades. Este soporte telemático es particularmente útil para conectar virtualmente 
al docente y al estudiante (García, 2018).

En esta investigación se trabaja con un Sistema de Gestión de Contenido Web, el cual es definido por el Centro de Apoyo Tecnológico a Emprendedores, Fundación Parque Científico y Tecnológico de Albacete (2012) como una herramienta que permite crear, editar, modificar y publicar contenidos Web, especialmente portales Web, ya estén dirigidos a una audiencia interna o externa.

Clasificación de los gestores de contenidos según su funcionalidad

Una de las clasificaciones de los gestores de contenido, la cual es tomada para esta investigación es según su funcionalidad, a saber, se clasifican en: Blogs, wikis, plataformas de enseñanza, publicaciones digitales y redes sociales; a continuación, se describen cada uno de ellos (García, 2018).

\section{Blogs}

Un blog es un sitio web propio creado por uno o varios autores donde periódicamente publican y comparten con otros usuarios de la red una serie de artículos, también llamados entradas o posts. Su éxito radica en el hecho de poder publicar información de una manera rápida y fácil gracias a plataformas de gestión de contenidos para la creación de blogs. Además, admiten que los lectores visitantes puedan aportar sus comentarios. Son, por tanto, una potente herramienta para la publicación y comunicación que favorece el intercambio y el debate de ideas.

Actualmente, los blogs tienden a especializarse en temáticas muy concretas por lo que también resultan una potente herramienta para la búsqueda de información de nuevos conocimientos. La comunidad de blogs o blogosferas va en aumento en los aspectos sociales, culturales, políticos y educativos, ya que al tener los autores total independencia para escribir sobre lo que estimen oportuno en su propio blog, son un indicador de lo que realmente preocupa a la sociedad.

En la docencia universitaria al blog se lo puede usar como innovación educativa para complementar las clases tradicionales y fomentar el aprendizaje colaborativo (Duarte, 2015); pueden ser utilizados para promover el desarrollo de competencias socio-cognitivas básicas y competencias digitales necesarias para desempeñarse eficientemente en la Sociedad de Conocimiento. Por su parte Tamayo (2016) opina que los blogs brindan facilidades para la creación de contenidos y favorecer el aprendizaje.

Existen innumerables y distintos tipos de blogs, según Kronenberg (2019) pueden conocerse ciertas categorías que agrupan algunos tipos, pero no pueden asumirse como un factor definitivo o limitante, según este autor se encuentran blogs para hacer dinero, sobre tutoriales, sobre viajes, estilos de vida, de productos, entre otros. Estas ideas concuerdan con Equipo Editorial (2019), quienes afirman que puede hablarse de diversos tipos de blogs tales como: educativos, divulgativos, de ventas, de servicio, o pueden ser clasificados según sus funciones y temáticas en cuatro tipos: personal, profesional, corporativo y temático o de nicho.

En relación con la tipología de blogs, Mejía (2017), presenta y describe ocho tipos, entre los cuales se encuentran: los personales, microblogging, corporativos y organizacionales, 
educativos, de género, por el tipo de medio de comunicación pueden ser: video blog, fotoblog, scketchblog, thumbleblogs; por el tipo de dispositivo de acceso pueden ser: moblog, wearable wireless webcam y el blog inverso o de varios usuarios. Otro autor que presenta una descripción de cuatro grandes tipos (por autoría, tipo de contenido, formato y fórmula de gestión) integrados por otros tipos de blogs es Bruguera (s/f), la cual se puede observar en el Tabla 1.

Tabla 1

Tipos de blogs.

POR AUTORÍA

Blog individual: el caso más clásico de diario personal en línea.

Cuantitativa

Blog colectivo: La autoría corresponde a un conjunto de personas particulares identificadas explícitamente o que mantienen una identidad simbólica como conjunto.

Cualitativa

Blog personal: de autoría libre e independiente por parte de usuarios particulares individuales o colectivos identificados o bajo seudónimo grupal.

Blog corporativo: impulsado individualmente o colectivamente desde el seno de empresas, instituciones u otro tipo de organización, como complemento más o menos informal de sus estrategias comunicativas.

- En el ámbito interno, de comunicación y circulación de información y relación entre personas del interior de la organización.

- En el ámbito externo, de proyección de contenidos y flujo comunicativo hacia ámbitos y escenarios ajenos a la organización.

\section{POR TIPO DE CONTENIDO}

Direccionamiento de enlaces

Información

personal

Circulación de noticias
El tipo de blog originario, en el cual el autor se centra sobre todo en proponer y comentar enlaces a otros sitios que consideraba interesantes, planteando así su blog como un directorio comentado de hipervínculos.

El blog personal clásico, centrado en contenidos y reflexiones que el autor considera de interés publicar en cada momento.

Blog centrado en la recomendación, ampliación y comentario de hechos noticiables desde el punto de vista del autor y de las respuestas de los lectores. 


\section{Temáticas}

especializadas

\section{POR FORMATO}

\section{Basados en texto}

Basados en imagen

Basados en sonido

\section{POR FÓRMULA DE GESTIÓN}

Propia

En línea
Propia: incluye los blogs, cuya gestión de contenidos se hace desde

Con diferentes modalidades híbridas de las categorías anteriores recomendación de enlaces, reflexión personal, comentario de noticias... también pueden ser clasificados según su ámbito temático de referencia o especialización, como de tecnología, religión, ecología, política, economía, literatura...

Publicación, circulación e intercambio de anotaciones textuales redactadas. Constituyen la mayor parte del universo de blogs.

Fotografía. En los denominados fotoblogs la circulación de información se basa en fotografías e imágenes digitales, no en el texto redactado. Sitios como Fotolog (inglés) y Fotoblog (castellano) indexan abundantes ejemplos.

Vídeo. El videoblog -o vblog- el flujo comunicativo se basa en el vídeo y la imagen en movimiento. El tamaño de las secuencias digitales todavía origina más problemas de alojamiento que las fotografías, por lo cual hay que buscar la circulación comunicativa sobre vídeo en sitios como YouTube, Google Video, Yahoo! Video o MSN Video, superficies virtuales de intercambio comunicativo que, sin generar exactamente videoblogs, abren un animado fenómeno de comunicación entre usuarios a partir de la imagen en movimiento.

Los audioblocs o podcasts son blogs en los que se intercambian ficheros de sonido y audio herramientas y programas instalados en el computador del autor. El alojamiento del blog en estos casos suele realizarse en un servidor corporativo o contratado directamente por el usuario particular.

- Cuando el autor del blog utiliza uno de los numerosos servicios gratuitos ofrecidos de forma conjunta alojamiento y herramientas de mantenimiento y gestión del blog.

- Cuando el autor del blog puede gestionar, mantener y actualizar los contenidos desde dispositivos portátiles como los teléfonos móviles, las PDA (Personal Digital Assistant) o los PocketPC. Se conoce como blog móvil o moblog.

Fuente: Nivela (2020) a partir de Bruguera (s/f). 


\section{Wikis}

Una wiki es un sitio web cuyas páginas pueden ser editadas por múltiples usuarios a través del navegador (Díez et al., 2017). En una wiki cualquier usuario puede acceder a la edición de los bloques de contenidos y participar en la discusión comentando dichos bloques, lo que lo hace vulnerable en cuanto a la seguridad y garantía de la veracidad de la información se refiere. Los sitios de alojamiento web más utilizados en el área de la educación para la creación de wikis son: PBworks, Wetpaint, Wikia, Google Sites y Wikispaces.

Las wikis aplicadas en docencia superior, permiten a los estudiantes mostrar los logros alcanzados a lo largo de su proceso de aprendizaje. Además contribuyen favorablemente al proceso educativo, desempeñando una serie de funciones tales como: la motivación y persuasión del estudiante para realizar actividades de la asignatura, el apoyo al aprendizaje autónomo, el establecimiento de una vía de comunicación directa entre el profesor y los alumnos fuera del entorno del aula y la evaluación formativa (Ortega. García y Díez, 2016). Por su parte Núñez, Míguez y Seoane (2016), consideran de utilidad proyectar el uso de wikis en los cursos educativos para favorecer el proceso de enseñanza aprendizaje.

\section{Plataformas de enseñanza}

Actualmente, a diferencia de la formación tradicional presencial, existen otras herramientas como son las plataformas de enseñanza, las cuales mediante la teleformación aportan otras modalidades de enseñanza. Según Viñas (2017), estas modalidades, según su uso se pueden clasificar en: B-learning, Elearning, W-learning, T-learning y M-learning. A continuación se esbozan cada una de ellas.
- B-learning: es una herramienta que sirve como apoyo a la enseñanza presencial, combina la enseñanza presencial con la tecnología no presencial.

- E-learning: es la plataforma que concibe la formación totalmente a distancia, apoyada en el uso de tecnologías basadas en Internet para proporcionar soluciones para la adquisición de conocimiento y habilidades o capacidades.

- M-learning: consiste en la plataforma que concibe el aprendizaje móvil, para la formación a distancia con el uso de tecnologías móviles como smartphone, tablets, lectores de MP3, ipad, entre otros.

- T-learning: es un sistema de aprendizaje transformativo, en el cual el uso de las tecnologías es parte del proceso, del desarrollo de contenidos y actividades que pueden darse de forma presencial o virtual. Se focaliza en el desarrollo de las habilidades en el hacer del estudiante.

- W-learning: constituye la plataforma de formación a distancia cooperativa, con base en herramientas colaborativas de la web.

El uso pedagógico de plataformas o mundos virtuales, según Monterroso (2011) puede contribuir a disminuir las sensaciones de soledad e incomunicación que pueden experimentar los estudiantes a distancia durante su proceso de aprendizaje. Además, se pueden realizar actividades de aprendizaje participativas y constructivas. Entre las aplicaciones educativas cabe destacar la realización de simulaciones, colaboraciones, juego de roles, trabajar en clases virtuales, construir ambientes colaborativamente entre otros. 


\section{Publicaciones digitales}

Son obras a las que se accede mediante algún dispositivo tecnológico electrónico y que, además de textos, pueden reproducir imágenes, audio y video. El surgimiento se da gracias a la masificación de las computadoras personales y de la conexión a internet. Entre las publicaciones digitales se encuentran: los libros electrónicos o ebooks, las obras de consulta, tales como diccionarios, manuales y enciclopedias; las publicaciones periódicas: revistas, revistas académicas especializadas, periódicos, etc.; las bibliotecas digitales; Coursepacks o dossier electrónico, los cuales son los materiales de lectura que provee el profesor para sus estudiantes en una asignatura y los catálogos (Tavares, 2017).

Pueden clasificarse en dos tipos: fijas y en línea. Las fijas son aquellas que se graban en algún soporte portátil de almacenamiento: CD, DVD, pen drive, entre otros. Las publicaciones en línea, están conformadas por aquellas que se pueden consultar a través de internet, cuyos archivos digitales se encuentran físicamente dentro de una computadora o servidor, quien provee un espacio en su disco duro para ser compartidos en la red. Su difusión es mucho mayor cuando se encuentra en línea que cuando está en un soporte físico transportable.

Las publicaciones digitales como soportes electrónicos de apoyo a la docencia facilitan la presentación y el acceso al conocimiento dentro y fuera del aula; promueven la práctica del aprendizaje colaborativo y cooperativo; operan a nivel cognitivo en la edificación de nuevos saberes y destrezas técnicas (Monterroso, 2011).

\section{Redes sociales}

Las redes sociales se tratan de espacios virtuales en los cuales las personas de todo el mundo pueden expresarse y sobre todo comunicarse, sin importar en qué lugar del planeta se encuentren. El término red social o comunidad virtual, consiste en un servicio que se brinda a través de un sitio web, donde se reúne la gente para hablar, compartir ideas e intereses, hacer nuevos amigos o expresar opiniones; es decir, para comunicarse. Son un medio de comunicación social, que por el contrario de los medios tradicionales, cuyas plataformas son limitadas en cuanto a la cantidad de gente que puede interactuar al mismo tiempo, constituyen sitios cuyo contenido es diariamente publicado por millones de personas (Tecnología Informática, 2019).

Son una de las estructuras sociales más poderosas e innovadoras de la Web para el trabajo colaborativo en Red, que pueden convertirse en comunidades de aprendizaje o en redes de conocimiento en tiempo real y sin importar las distancias geográficas. Su naturaleza social, interactiva y comunicativa, principalmente de Facebook, Twitter, Instagram, WhatsApp, Edmodo, Linkedin, Google+, YouTube, Pinterest, Snapchat, entre otras y su gran popularidad entre los jóvenes estudiantes, hace que estas tecnologías puedan ser utilizadas como recurso educativo (González y Muñoz, 2016 y Prieto, 2016).

En Ecuador, según Del Alcázar (2019), se evidencia un crecimiento importante para el caso de Instagram y Linkedin, manteniéndose Facebook como la red social predominante, la Figura 1, muestra la cantidad de usuarios de las redes sociales en este país. 


\section{Figura 1}

Cantidad de usuarios de las redes sociales en Ecuador.



Fuente: (Del Alcázar, 2019).

El impulso del uso de las TIC en los procesos de enseñanza y aprendizaje ha transformado los modelos educativos con el empleo de contenidos didácticos digitales flexibles que ofrecen múltiples ventajas al incentivar un aprendizaje más efectivo, sobre todo mediante el uso de los gestores de contenido como elementos de las TIC impulsores o generadores de impacto en la construcción de conocimientos. Jiménez (2011) opina que las redes sociales son plataformas simples, accesibles, que permiten organizar e intercambiar contenido de una manera eficaz, debido a que poseen funcionalidades básicas de mucha utilidad en el entorno educativo.

\section{Variable de estudio}

\section{Gestores de Contenidos web}

\section{Definición conceptual}

Gestores de Contenido Web, es definido por el Centro de Apoyo Tecnológico a Emprendedores, Fundación Parque Científico y Tecnológico de Albacete (2012, p. 8) como "una herramienta que permite crear, editar, modificar y publicar contenidos Web, especialmente portales Web, ya estén dirigidos a una audiencia interna o externa".

\section{Definición operacional}

Los gestores de contenido operacionalmente 
son medidos a través de un instrumento relacionado con la gestión de contenidos en blogs, wikis, plataformas educativas, publicaciones periódicas y redes sociales, desde el punto de vista de los estudiantes.

\section{REFERENTES METODOLÓGICOS}

El tipo de paradigma asumido en esta investigación es el Cuantitativo, el cual según Palella y Martins (2012, p. 40), "relega la subjetividad humana y busca la verificación empírica de los hechos y sus causas, con el objetivo de establecer leyes universales", en este sentido, la complejidad humana se transforma en variables, las cuales son cuantificadas y analizadas para calcular la probabilidad estadística de que algo ocurra. De esta forma asume la objetividad como vía para alcanzar el conocimiento.

En el caso de las ciencias sociales, según Hernández. Fernández y Baptista (2014), el paradigma cuantitativo parte de un mundo social ya conocido, el cual es externo al investigador y objetivo. Su marco referencial básico es el positivismo, su meta es generar teorías, a partir de comparar la investigación previa con los resultados del estudio aplicando la lógica deductiva. Particularmente, esta investigación parte de una realidad ya conocida y establecida como lo es el proceso educativo desarrollado en la carrera de Informática de la Universidad de Guayaquil, Facultad de Filosofía, Letras y Ciencias de la Educación.

\section{Tipo de investigación}

La investigación es de tipo descriptiva, con estas investigaciones:
Se busca especificar las propiedades, las características y los perfiles de personas, grupos, comunidades, procesos, objetos o cualquier otro fenómeno que se someta a un análisis. Es decir, únicamente pretenden medir o recoger información de manera independiente o conjunta sobre los conceptos o las variables a las que se refieren, esto es, su objetivo no es indicar cómo se relacionan éstas". (Hernández. Fernández y Baptista, 2014, p.92)

\section{Diseño de investigación}

El diseño asumido es no experimental, debido a que se observan los fenómenos tal como se dan en su contexto natural, para posteriormente analizarlos. Es sistemática y empírica, las variables no se manipulan. Las inferencias sobre las relaciones entre variables se realizan sin intervención o influencia directa, y dichas relaciones se observan tal como se dan en su contexto natural (Hernández. Fernández y Baptista, 2014).

En el contexto de esta investigación se observarán los fenómenos tal como se dan en su entorno natural, es decir, en el contexto educativo universitario de la universidad de Guayaquil, para posteriormente analizarlos según lo planteado en el objetivo. La variable en estudio, gestores de contenido web, ocurre y no es posible manipularla, no se tiene control directo, ni se puede influir sobre ella, porque ya sucedieron o están sucediendo, al igual que sus efectos. Fue transeccional o transversal, debido a que se recolectaron datos en un solo momento, específicamente durante el primer período del año 2020, en un tiempo único. Su propósito es describir variables y analizarla en un momento dado (Hurtado, 2012). 


\section{Población y muestra}

Para la presente investigación, la población se encuentra conformada por 150 estudiantes, pertenecientes a la Universidad de Guayaquil, Facultad de Filosofía, Letras y Ciencias de la Educación, carrera Informática. En esta se aplicó el muestreo no probabilístico por conveniencia o también llamado muestreo intencional, la cual selecciona los elementos con base en criterios o juicios previamente establecidos por el investigador (Arias, 2012). Los criterios de selección se presentan en la Tabla 2.

Tabla 2

Criterios de selección de la muestra para estudiantes

\begin{tabular}{ll}
\hline Criterios & Estudiantes \\
\hline Pertenecen a los cursos Diurnos o vespertino & $\mathrm{Si}$ \\
Pertenecen al primer nivel de la carrera informática & $\mathrm{Si}$ \\
Dispuestos a participar en la investigación & $\mathrm{Si}$ \\
Acceso por parte del investigador & $\mathrm{Si}$ \\
\hline
\end{tabular}

Fuente: Elaborado por autores.

En función de los criterios anteriormente descritos, se seleccionaron 50 estudiantes para la conformación de la Muestra.

\section{Técnicas e instrumentos de recolección de información}

En esta investigación se utilizó la técnica de la encuesta, la cual según Hernández. Fernández y Baptista, (2014), consiste en un conjunto de preguntas de una o más variables a medir o investigar. Como instrumentos de recolección de datos se usó el cuestionario.

El cuestionario elaborado para determinar la funcionalidad de diversos gestores de contenidos Web quedó estructurado en 5 ítems, correspondiendo a la Gestión de contenidos en blogs, wikis, plataformas educativas, publicaciones periódicas y redes sociales; los cuales tuvieron tres opciones en alternativas de respuesta que corresponden a la escala: Fácil (codificada con 3), ni fácil ni difícil (codificada con 2) y difícil (codificada con 1).

\section{Validez y confiabilidad}

Para esta investigación se utilizó el criterio de validez de expertos a través del juicio de cinco expertos en el área. Según Pardo y Ruíz (2005), la fiabilidad o confiabilidad es la capacidad del instrumento para medir de forma consistente, precisa y sin error la característica que se desea medir. Por su parte, Hernández. Fernández y Baptista, (2014) afirman que la confiabilidad cuantitativa se determina al calcular el coeficiente de fiabilidad.

El modelo de fiabilidad o confiabilidad seleccionado fue el Modelo Alfa de Cronbach, ya que, valora la consistencia interna de la escala a partir de la correlación inter-elementos promedios 
(Pardo y Ruíz, 2005). Éste método asume que el instrumento está compuesto por elementos homogéneos aleatoriamente seleccionados de la población que miden la misma característica. Para esto se aplicó una prueba piloto, que consistió en aplicar el instrumento a un pequeño grupo (10 elementos) con características similares a las de la muestra definitiva, para hacer las correcciones y ajustes necesarios. Para el cálculo de éste coeficiente se utilizó el Paquete estadístico SPSS y el baremo de interpretación presentado en el Tabla 3.

\section{Tabla 3.}

Baremo para la interpretación de la confiabilidad

\begin{tabular}{ll}
\hline Baremo & Interpretación \\
\hline $0,01-0,20$ & Muy Baja \\
$0,21-0,40$ & Baja \\
$0,41-0,60$ & Moderada \\
$0,61-0,80$ & Alta \\
$0,81-1,00$ & Muy Alta \\
\hline
\end{tabular}

Fuente: Hernández, Fernández y Baptista, (2014).

Luego de la aplicación de la prueba piloto y procesamiento de los datos se obtuvo un Alfa de Cronbach de 0,89 , siendo la confiabilidad muy alta según el baremo de la Tabla 3.

\section{ANÁLISIS DE DATOS}

En esta investigación con relación a su tipo y diseño, se asumió la estadística descriptiva. Una vez aplicado el instrumento de recolección de datos, se tabularon y se determinaron medidas de tendencia central (moda, media y mediana) y de dispersión (desviación estándar, puntaje mínimo y puntaje máximo), para la variable: Gestores de contenido, esto fue realizado a través del programa estadístico para ciencias sociales (SPSS), para su interpretación se utilizó el baremo de la Tabla 4

\section{Tabla 4.}

Baremo de interpretación de las medidas de tendencia central para los criterios de funcionalidad de gestión de contenidos

\begin{tabular}{ll}
\hline Categorías & Intervalo \\
\hline Muy baja & $0,01-0,60$ \\
Baja & $0,61-1,20$ \\
Media & $1,21-1,80$ \\
Alta & $1,81-2,40$ \\
Muy alta & $2,41-3,00$ \\
\hline
\end{tabular}

Fuente: Elaborado por autores.

\section{RESULTADOS}

A continuación se presenta la Tabla 5 con las estadísticas descriptivas de los datos obtenidos luego de la aplicación del cuestionario. 
Tabla 2

Estadísticas descriptivas de Gestión de contenidos en blogs, wikis, plataformas educativas, publicaciones periódicas y redes sociales.

\begin{tabular}{|c|c|c|c|c|c|}
\hline & $\begin{array}{l}\text { Gestión de } \\
\text { contenidos en } \\
\text { un Blogs }\end{array}$ & $\begin{array}{l}\text { Gestión de } \\
\text { contenidos en } \\
\text { Wikis }\end{array}$ & $\begin{array}{l}\text { Gestión de } \\
\text { contenidos en } \\
\text { Plataformas } \\
\text { educativas }\end{array}$ & $\begin{array}{l}\text { Gestión de } \\
\text { contenidos en } \\
\text { Publicaciones } \\
\text { periódicas }\end{array}$ & $\begin{array}{l}\text { Gestión de } \\
\text { contenidos en } \\
\text { Redes sociales }\end{array}$ \\
\hline Media & 2,64 & 2,18 & 2,55 & 1,55 & 2,64 \\
\hline Moda & 3 & 2 & 3 & 1 & 3 \\
\hline Mediana & 3 & 2 & 3 & 1 & 3 \\
\hline $\begin{array}{l}\text { Desviación } \\
\text { Estándar }\end{array}$ & 0,50 & 0,60 & 0,52 & 0,82 & 0,50 \\
\hline Máximo & 3 & 3 & 3 & 3 & 3 \\
\hline Mínimo & 2 & 1 & 2 & 1 & 2 \\
\hline Rango & 1 & 2 & 1 & 2 & 1 \\
\hline $\begin{array}{l}\text { Categoría de } \\
\text { la media }\end{array}$ & Muy alta & Alta & Muy alta & Media & Muy alta \\
\hline $\begin{array}{l}\text { Categoría de } \\
\text { la desviación } \\
\text { estándar }\end{array}$ & Muy baja & Muy baja & Muy baja & Baja & Muy baja \\
\hline
\end{tabular}

Fuente: Elaborado por autores.

La Tabla 5 evidencia que la media para la gestión de contenidos en blogs, es 2,64, lo que indica que es muy alta, la moda o valor que más se repite y mediana o valor que se encuentra en la mitad coincidieron en el caso de los blogs con 3, interpretadas como muy altas. La desviación estándar fue de 0,50 que indica una dispersión muy baja de los puntajes. El mínimo se ubicó en 2 (alta) y el máximo en 3 (muy alta). Asimismo, en la gestión de contenidos en wikis, la media fue de 2,18 (alta), la moda de 2 y la mediana de 2, interpretadas como altas. La desviación fue de 0,60 que indica una dispersión muy baja de los puntajes. El mínimo se ubicó en 1 (baja) y el máximo en 3 (muy alta).
De igual forma, para la gestión de contenidos en plataformas educativas, la media fue de 2,55, interpretada como muy alta, la moda de 3 o muy alta y la mediana de 3 o muy alta. La desviación fue de 0,52 que indica una dispersión muy baja de los puntajes. El mínimo se ubicó en 2 (alta) y el máximo en 3 (muy alta). Con relación a la gestión de contenidos en publicaciones periódicas la media fue de 1,55 , interpretada como media, la moda de 1 o baja al igual que la mediana 1 o baja. La desviación fue de 0,82 que indica una dispersión baja de los puntajes. El mínimo se ubicó en 1 (baja) y el máximo en 3 (muy alta).

La media para la gestión de contenidos en redes 
$\begin{array}{lllllll}R & \text { E } & \text { V } & \text { I } & S & T & A\end{array}$

Public a n d o

I S S N $133900-9304$

\section{CONCLUSIÓN}

La funcionalidad de diversos gestores de contenidos Web aplicados en la práctica educativa de estudiantes universitarios tiende a ser muy alta en el caso de la creación, gestión, publicación y presentación de contenidos en blogs y redes sociales, con relación a las plataformas educativas los aprendices opinan que sólo la presentación de contenidos en ellas tiene una funcionalidad muy alta; la creación, gestión y publicación de contenidos en esta y en wikis y publicaciones periódicas es considerada alta. Es decir, en cuanto a funcionalidad prefieren blogs y redes sociales.
Este hallazgo apoya las ideas de Duarte (2015), quien opina que al blog se lo puede usar para fomentar el aprendizaje colaborativo; además pueden ser utilizados para promover el desarrollo de competencias socio-cognitivas básicas y competencias digitales necesarias para desempeñarse eficientemente en la Sociedad del Conocimiento. También concuerdan con lo expuesto por Tamayo (2016), quien opina que los blogs brindan facilidades para la creación de contenidos y favorecer el aprendizaje.

Con relación a la funcionalidad de las redes sociales, concuerda con Jiménez (2011) quien opina que las redes sociales son plataformas simples, accesibles, que permiten organizar e intercambiar contenido de una manera eficaz, debida a que poseen funcionalidades básicas de mucha utilidad en el entorno educativo.

En cuanto a las publicaciones periódicas digitales como soportes electrónicos de apoyo a la educación, según Monterroso (2011), se tiene que estas facilitan la presentación y el acceso al conocimiento dentro y fuera del aula; promueven la práctica del aprendizaje colaborativo $y$ cooperativo; operan a nivel cognitivo en la edificación de nuevos saberes y destrezas técnicas. 


\section{REFERENCIAS}

Arias, F. (2012). El Proyecto de Investigación. Introducción a la metodología científica. Sexta Edición. Caracas, Venezuela: Editorial Episteme.

Bruguera, E. (Sin fecha). ¿Qué es un blog? España, Universitat Oberta de Catalunya. Recuperado de http://openaccess.uoc.edu/ webapps/o2/bitstream/10609/17821/5/ XX08_93006_01331-3.pdf.

Celi, L. (2018). Glosario de Términos de las actividades de I+D+I. Quito, Ecuador: Unidad de Difusión $\mathrm{I}+\mathrm{D}+\mathrm{i}+\mathrm{V}$.

Centro de Apoyo Tecnológico a Emprendedores, Fundación Parque Científico y Tecnológico de Albacete (2012). Estudio de los Sistemas de Gestión de Contenidos Web. Castilla-La Mancha. Recuperado de https://www.bilib.es/fileadmin/ estudio_sistemas_gestion_contenidos_we b_cms.pdf

Del Alcázar, J. (01 de enero de 2019). Estado Digital Ecuador 2019-Estadísticas Digitales Actualizadas. Formación Gerencial. [Publicación de blog]. Recuperado de https:// blog.formaciongerencial.com/ es t a d odigitalecuador/ \#Usuarios_de_Redes_Sociales_en_Ecuador.

Delgado, M. (2018). Hacia la transformación en educación superior: investigación científica y tecnológica e innovación en América Latina y el Caribe. Revista Educación Superior y Sociedad: La CRES 2018: una discusión en línea. 25(25), 89-109.
Duarte, P. (2015). The Use of a Group Blogto Actively Support Learning Activities. Active Learning in Higher Education. 16(2), 103-117.

García, A. (2018). Canales de distribución y publicación utilizados en los sistemas gestores de información. Barcelona, España: Editorial Elearning.

González, C. y Muñoz, L. (2016). Redes Sociales su impacto en la Educación Superior: Caso de estudio Universidad Tecnológica de Panamá. Revista Campus Virtuales. 5(1), 84-90.

Hernández, R.; Fernández, C. y Baptista, M. (2014). Metodología de la Investigación. México, México: McGraw-Hill Education.

Hurtado, J. (2012). Metodología de la Investigación. Caracas, Venezuela: Editorial Quirón.

Jiménez, R. (31 de enero de 2011). El papel de las redes sociales en la educación. (Mensaje en un blog). Educaweb. Recuperado de https:// www.educaweb.com/noticia/2011/01/31/ papel-redes-sociales-educacion-4577/.

Kronenberg, I. (2019). Blogging: La mejor pequeña guía para administrar un blog redituable. España: Babelcube Inc.

Mejía, J. (2017). Mercadotecnia Digital: Una descripción de las herramientas que apoyan la planeación estratégica de toda innovación de campaña web. México, México: Grupo Editorial Patria.

Monterroso, E. (2011). Educación inmersiva: educación práctica del derecho en 3D. Revista Icono 14. 2(9) 84-100. 


\section{R E V I S T A \\ Public a n d o \\ I S S N $133900-9304$}

Núñez, I., Míguez, M., \& Seoane, G. (2016). Wikis en Moodle: la mirada de estudiantes y docentes. Educación química, 27(4), 257-263.

Ortega, M.; García, P. y Díez, A. (2016). El proceso de la innovación en el contexto de la educación superior universitaria. Madrid, España: Editorial LDL.

Palella, S. y Martins, F. (2012). Metodología de la investigación cuantitativa. Caracas, Venezuela: Editorial FEDUPEL.

Pardo, A. y Ruíz, M. (2005). Análisis de Datos con SPSS 13 Base. Madrid, España: Editorial Mc Graw Hill.

Prieto, J. (2016). Una aproximación metodológica al uso de redes sociales en ambientes virtuales de aprendizaje para el fortalecimiento de las competencias transversales de la Universidad EAN. Revista Virtu@lmente. 1(1), 1-16.

Tamayo, Y. (2016). Los Blogs educativos como recurso didáctico en el proceso de enseñanzaaprendizaje de la historia. Boletín Virtual. 5, 1-9. Recuperado de https://dialnet.unirioja.es/ descarga/articulo/6064932.pdf.

Tavares, R. (19 de febrero de 2017). Procesos editoriales. Fundamentos de la producción editorial de publicaciones unitarias, periódicas y digitales. [Publicación de blog]. Recuperado de https:// procesoseditoriales.blogs.sapo.pt/capitulo-4publicaciones-digitales-1407.

Tecnología Informática. (2019). Redes Sociales: Definición, historia y tipos de redes. Recuperado de https:// tecnologia-informatica.com/redessociales-definicion-historia/.

\section{sociales-definicion-historial.}

Viñas, M. (2017). La importancia del uso de plataformas educativas. Revista Letras. 6, 157-169. 